\title{
Comparative Study of LBPH and Haar features in Real Time Recognition Under Varying Light Intensities
}

\author{
Dennies Rocky, Aajin Roy, Anujith S, Eldho K Paul, Akas G Kamal
}

\begin{abstract}
Face recognition is a commonly used biometric and has a wide range of applications. We used an access control system that integrates face recognition technology. This paper discusses two algorithms that have been used in the face detection, Haar features and Local Binary Patterns Histogram $(\mathrm{LBPH})$. The experimental set up is done in an open environment using OpenCV library. Comparative study has been made between these two algorithms based on parameters like illumination and hit rate. For the testing, the same training set and samples were used.
\end{abstract}

Keywords : Face recognition, Haar features, $\mathrm{LBPH}$, OpenCV

\section{INTRODUCTION}

Face is unique property of human beings which is a primary focus of attention in social life playing an important role in conveying identity and emotions. This unique property is quite robust despite large variations in visual stimulus due to changing conditions such as environment, aging and other natural factors (beards, moustache, hairstyles and spectacles). Human's capabilities are very good at recognizing and remembering faces despite the passage of time. Hence, it is essentially beneficial if the current computer technologies become robust as humans in face detections.

Human beings perform face recognition automatically every day and practically with no effort. Although it sounds like a very simple task for a computer, it has many variables that can impair the accuracy of the methods, for example: illumination variation, low resolution and occlusion.

Face detection is a computer coding technology that determines the location and size of human faces in a given image format. It detects only the facial features and ignores the rest. Here two algorithms, Haar cascades and Local Binary Pattern Histogram (LBPH) were evaluated based on

Revised Manuscript Received on July 22, 2019.

* Correspondence Author

Dennies Rockky*, Electronics and Communication, Adi Shankara Institute of Engineering and Technology, Kalady, India. Email: denniesrocky007@gmail.com

Aajin Roy, Electronics and Communication, Adi Shankara Institute of Engineering and Technology, Kalady, India. Email: aajinroy09@gmail.com

Eldho K Paul, Electronics and Communication, Adi Shankara Institute of Engineering and Technology, Kalady, India. Email: eldhokpau198@ gmail.com Anujith S, Electronics and Communication, Adi Shankara Institute of Engineering and Technology, Kalady, India. Email: gr8anujith@gmail.com

Akas G Kamal, Electronics and Communication, Adi Shankara Institute of Engineering and Technology, Kalady, India. Email: akas.ec@adishankara.ac.in some parameters ${ }^{[3]}$. In our simulations in OpenCV, the examples shown are real time and images are captured by using an USB camera.

\section{METHOD}

Two algorithms evaluated here are Local Binary Pattern Histogram (LBPH) and Haar cascades. Each algorithm follows different procedures in detecting the face. There are many variables that impair the accuracy of the methods. A detailed description about the two methods are discussed in the following upcoming sections.

\section{A. Local Binary Pattern Histogram}

Local Binary Pattern Histogram (LBPH) is a simple yet very efficient texture operator which labels the pixels of an image by thresholding the neighborhood of each pixel and considers the result as a binary number ${ }^{[2]}$. LBPH extracts local features in the face and match it with the most similar face image in the database. LBPH is a method that works by dividing the face image into several blocks. Histograms will be calculated for each block and in the matrix we compare the pixels with the centre pixel. At the end we will get a binary number which will be converted to decimal format ${ }^{[7]}$. It will be combined together under one vector which will help to recognize the face in the database.

To understand more about Local Binary Pattern algorithm, consider the following example.

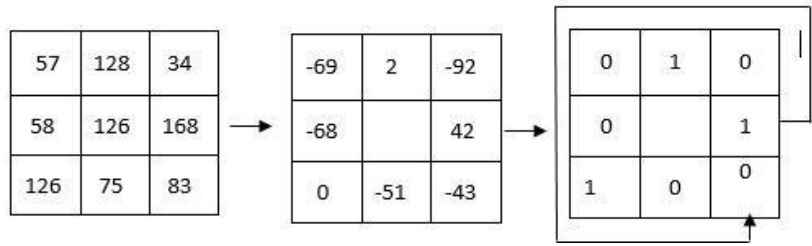

Figure 1: The basic LBPH operation ${ }^{[9]}$.

The original LBPH operator labels the pixels of an image by keeping the $3 \times 3$ neighborhood or it can be also said as a matrix. Each pixel has value which can vary depending upon the image and pixel quality.

If middle pixel " 126 " is chosen which have eight neighbor, subtract these neighbor values with 126 if the value is less than zero put it as zero and if the value is more than zero put it as one. Following the arrow direction in the figure 1.1, you will get the binary number as 10100100 i.e.

164 in decimal system. The overall resulting LBPH can be expressed by the equation (1), 


\section{LBPH $(\mathrm{Xc}, \mathrm{Yc})=\sum^{7} s($ in-ic $) 2^{n}$}

where $\mathrm{n}$ runs over the eight neighbour of the center pixel, ic and in are gray-level values of the central pixel and the function $\mathrm{s}(\mathrm{x})$ is defined in (2).

$$
\begin{aligned}
& s(x)=\{1, x>=0 \\
& \{0, x<0
\end{aligned}
$$

But there are some limitations to basic LBPH operator that its small $3 \times 3$ matrix method cannot capture the dominant features with large scale structures. As a result, to deal with the texture at different scales, the operator can be extended to use neighborhoods of different size.

\section{B. Haar}

Object detection using Haar feature based cascade classifiers is an effective object detection method proposed by Paul Viola and Michael Jones ${ }^{[1]}$. It is a machine learning based approach where a cascade function is trained from a lot of positive and negative images. It is then used to detect objects in other images. Here, we are working with face detection. Initially, the algorithm needs a lot of positive images (images of faces) and negative images (images without faces) to train the classifier. Then we need to extract features from it. Features are nothing but numerical information extracted from the images that can be used to distinguish one image from another ${ }^{[4]}$. Figure 2 shows different Haar features.
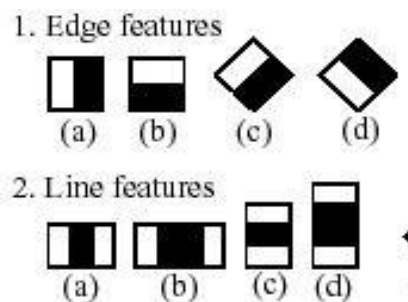

3. Center-surround features

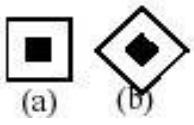

Figure 2: Haar-like features ${ }^{[6]}$

A simple rectangular Haar like feature can be defined as he differences of the sum of pixels of areas inside the rectangle, which can be at any position and scale within the original image. A Haar-like feature considers neighboring rectangular regions at a specific location in a detection window, sums up the pixel intensities in each region and calculates the difference between these sums. First, the pixel values inside the black area are added together then the values in the white areas are summed.

This result is used to categorize image sub regions. An example of this would be the detection of human face. Commonly the areas around the eyes are darker than the areas on the cheeks ${ }^{[5]}$. One example of a Haar like feature for face detection is therefore a set of two neighboring rectangular areas above the eye and cheek region.

\section{EXPERIMENT}

We compared two algorithms, Local binary pattern and Haar like feature using OpenCV in Raspberry Pi based on parameters like hit rate, detection rate, distance, illumination, pose variation and detection speed.

Initially, we captured images of 40 individuals in ten different postures and created a database (Figure 3). Each individual was given a unique id which corresponds to an image in the trainer set.

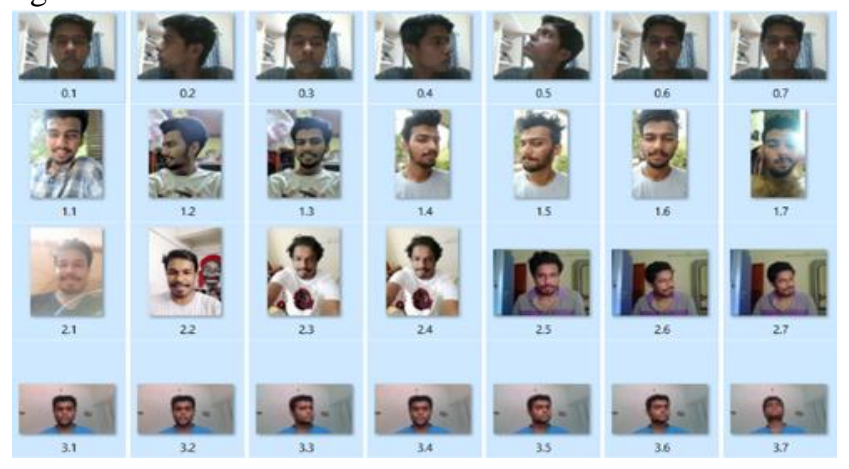

Figure 3: Database.

Then real time image of an individual was captured and compared with different images in the trainer. One with greater similarity will be matched with the captured image. This is done with the help of the detection algorithms Local Binary Pattern and Haar.

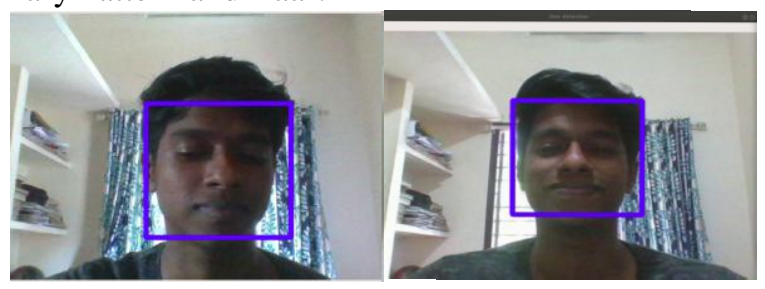

Figure 4: Face detection using Haar in various light intensities.

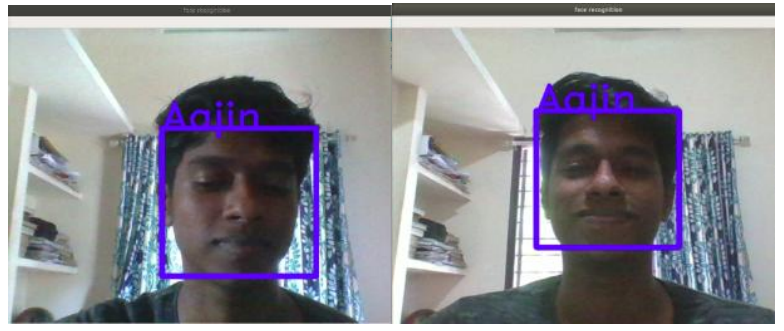

Figure 5: Face detection using LBPH in various light intensities

\section{RESULT AND DISCUSSION}

The algorithms were tested on Raspberry Pi 2 model B with a Quad core (900MHz), 1GB SDRAM running Debian Jessie using OpenCV library based on the parameters like hit rate, detection rate, pose variation, illumination. The camera being used is a Logitech C310 Webcam with a 720p maximum resolution.

Table I: Haar result

\begin{tabular}{|c|c|c|}
\hline Light Intensity(lux) & Detected faces & Hit Rate \\
\hline $25-133$ & $56 / 200$ & $28.0 \%$ \\
\hline $110-150$ & $108 / 200$ & $54 \%$ \\
\hline $150-213$ & $115 / 200$ & $57.5 \%$ \\
\hline$>213$ & $178 / 200$ & $89.0 \%$ \\
\hline
\end{tabular}

Table II: LBPH result 


\begin{tabular}{|c|c|c|}
\hline Light Intensity(lux) & Detected faces & Hit Rate \\
\hline $25-133$ & $25 / 200$ & $12.5 \%$ \\
\hline $110-150$ & $77 / 200$ & $38.5 \%$ \\
\hline $150-213$ & $112 / 200$ & $56.0 \%$ \\
\hline$>213$ & $152 / 200$ & $76.0 \%$ \\
\hline
\end{tabular}

\section{REFERENCES}

1. Paul Viola and Michael Jones, "Rapid Object Detection using a Boosted Cascade of Simple Features",IEEE Computer Society Conference on Computer Vision and Pattern Recognition 2001.

2. Snehal Humne and Prachi Sorte, "A Review on Face Recognition using Local Binary Pattern Algorithm”, International Research Journal of Engineering and Technology (IRJET), Volume: 05 Issue: 06, June-2018.

After the simulations of two algorithms, Haar and LBPH 3. Kushsairy Kadir, Mohd Khairi Kamaruddin, Haidawati Nasir, Sairul I Safie using Opencv in Raspberry $\mathrm{Pi}$, it has been found that Haar is more accurate than the LBPH. Table I and Table II shows the results of Haar and LBPH algorithms tested based on certain parameters. From the above results it shows that the hit rate of Haar was $89 \%$ and LBPH was $76 \%$ at optimum lighting condition. Both the algorithms had a low hit rate in case of multiple face detection depending on the number of faces in the image.

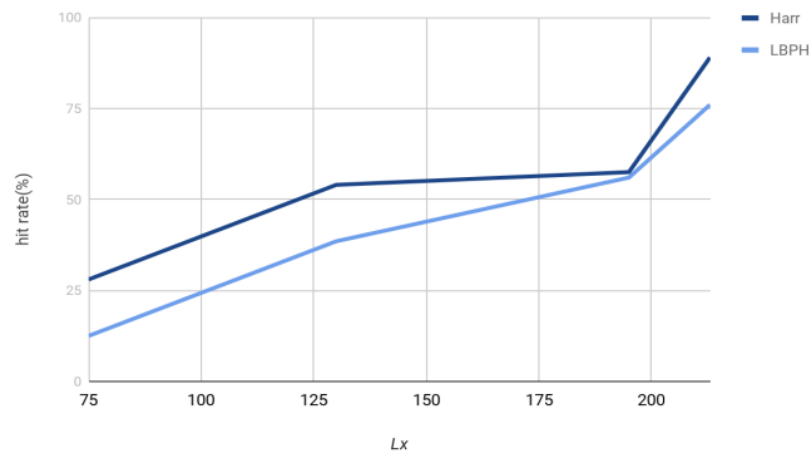
and Zulkifli Abdul Kadir Bakti, "A Comparative Study between LBP and Haar-like features for Face Detection Using OpenCV ”,International Conference on Engineering Technology and Technopreneurship(ICE2T) 2014.

4. Songyan Ma and Lu Bai, “ A face detection algorithm based on Adaboost and new Haar like features", International Conference on Software Engineering and Service Science (ICSESS), 2016.

5. R. Padilla, C. F. F. Costa Filho and M. G. F. Costa, "Evaluation of Haar Cascade Classifiers Designed for Face Detection" ,International Journal of Computer, Electrical, Automation, Control and Information Engineering Vol:6, No:4, 2012.

6. Jie Zhu, Zhiqian Chen, "Real Time Face Detection System using Adaboost and Haar like Features "International; Conference on Information Science and Control Engineering, April 2015.

7. Ahonen, Timo, Abdenour Hadid, and Matti Pietikainen. "Face description with local binary patterns: Application to face recognition." IEEE transactions on pattern analysis and machine intelligence, 2006.

8. Özdil A. and Özbilen M. M., "A Survey on Comparison of Face Recognition Algorithms", Application of Information and Communication Technologies (AICT), IEEE 8th International Conference, 2014.

9. J. Chao W L, Ding J J, Liu J Z. "Facial expression recognition based on improved local binary pattern and class-regularized locality preserving projection". Signal Processing, 2015.

10.Zheng Xiang, Hengliang Tan, Wienling Ye. "The excellent properties of dense gird-based HOG features on face recognition compare to gabor and LBP”,LNCS3021,2018.

Figure 6: comparison of haar and LBPH in different light intensity

As the light intensity increases the detection rate was increasing. If the light intensity is greater than $213 \mathrm{~lx}$, we get maximum hit rate. The result from the tables shows that Haar is more robust than LBPH based on parameters such as such as distance and illumination. The percentage of face detection using Haar was almost $13 \%$ more than LBPH algorithm. Under pose variation, the success rate of face detection was low in case of both the algorithms. The detection speed of LBPH was more than that of Haar by $314 \mathrm{~ms}$. The individual detection speed of both algorithms mainly depended on the size of the database.

Both the algorithms do not have any detection problem with racial faces, hairy faces or faces with glasses. Based on the results of the experiment, the Haar face detection algorithm is more reliable to detect the faces than LBPH even though it is a bit slower when compared to LBPH.

\section{CONCLUSION}

This paper discusses two methods or algorithms that have been used in face detection, Haar features and Local Binary Patterns Histogram (LBPH). Comparative study has been made between these two algorithms. Based on overall results, it can be concluded that LBPH has overall faster detection speed compared to Haar. But Haar is more robust than LBPH based on parameters such as such as distance and illumination. The percentage of face detection using Haar was almost $13 \%$ more than LBPH algorithm. From here, we can say that the Haar face detection algorithm is more reliable to detect the faces.
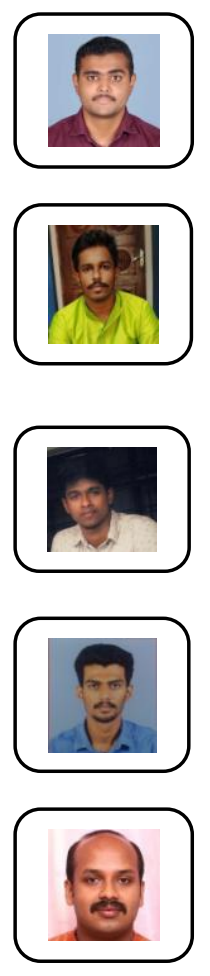

kas G Kamal working as Asst. Professor in the Departmen of Electronics \& Communication at Adi Shankara Institute of Engineering \& Technology, Kalady. He received his B.Tech in Electronics and Communications from CUSAT and completed his M.Tech in Advanced Digital Communication from MG University. He is having 13 years of teaching experience. He has published 7 national and international journals. 